\section{Una aproximación a la arquitectura de Richard Neutra desde su empatía con el usuario en el proceso de proyecto}

An approach to the architecture of Richard Neutra from his empathy with the user in the project process

Uma abordagem da arquitetura de Richard Neutra a partir de sua empatia com o usuário no processo de projeto

DOI: https://doi.org/10.18861/ania.2021.11.2.3177

\section{Arq. Raquel Cabrersidad de Valladolid}

Espana

hola@raquelcabrero.com

Recibido: $17 / 11 / 2021$
El artículo presenta una aproximación a la obra del arquitecto Richard Neutra analizando y poniendo en valor la empatía con el usuario, así como las acciones realizadas durante el proceso de proyecto orientadas a conocer y satisfacer las necesidades del mismo. Aunque en el campo del diseño es habitual contar con técnicas de empatía que se trabajan de manera consciente en arquitectura se ha venido haciendo de una manera más subconsciente. Debido a ello la exploración sobre el usuario ha quedado implíctamente integrada y, por tanto, diluida en una investigacion inicial más amplia, vinculada garantizar la funcionalidad del programa. La obra de Richard Neutra se presenta como adecuada para realizar este estudio, por el foco constante que el arquitecto pone en buscar el bienestar de las personas a través de los espacios habitados que diseña. La investigación se estructura en tres partes diferenciadas: la atención hacia las dimensiones fisiológica y psicológica de la persona; el análisis de la relación que se establece entre la persona y la naturaleza a través de su arquitectura; y la presentación de ejemplos específicos de técnicas aplicadas por el arquitecto para conocer al usuario, su estructura familiar y sus necesidades. A través de los casos estudiados y con apoyo de feedback específico de algunos de sus clientes, se confirm la fuerte orientación hacia el usuario en la arquitectura de Neutra, buscando garantizar su bienestar físico y emocional a través de la configuración de los espacios, así como la expansió del ser mediante la conexión con la naturaleza. Un arquitecto adelantado a su tiempo, que construye un fuerte vínculo empático con sus usuarios y aplica una serie de técnicas de empatía que tendrán un largo desarrollo posterior dentro de lo que hoy se conoce como Design Thinking.

Palabras clave: Richard Neutra, biorrealismo, etnografía empatía, arquitectura centrada en el usuario, proceso de diseño humanismo, Case Study Houses, Casa Miller, Casa Bailey, Eames. Outdoor
The article presents an approach to the work of the architect Richard Neutra analyzing and valuing empathy with the user, as well as the actions carried out during the design process aimed at knowing and satisfying the user's needs. Although it is common to consciously use empathy techniques in the field of design it has been done in a more subconscious way in architecture. The exploration of the user has been implicitly integrated and therefore, diluted in a broader initial investigation, linked to guaranteeing the functionality of the program. The work of Richard Neutra is presented as adequate to carry out this study, due to the constant focus that the architect places on seeking the wellbeing of people through the inhabited spaces that he designs. The research is structured in three different parts: attention to the physiological and psychological dimensions of the person; analysis of the relationship established between the person and nature through his architecture; and presentation of specific examples of techniques applied by the architect to better know the user, their family structure and their needs. The strong orientation towards the user in Neutra's architecture is confirmed through the cases studied and with the support of specific feedback from some of his clients. He seeks to guarantee their physical and emotional wellbeing through the configuration of spaces, as well as the expansion of being through connection with nature. An architect ahead of his time, who builds a strong empathic bond with his users and applies a series of empathy techniques that will have a long subsequent development within what is now known as Design Thinking.

Keywords: Richard Neutra, biorrealism, ethnography, empathy, user-centred architecture, design thinking process, humanism, Case Study Houses, Miller House, Bailey House, Eames.
O artigo apresenta uma abordagem da obra do arquiteto Richard Neutra analisando evalorizando a empatia com o usuario, bem conhecer satisfazer sus necessidadis. Emborano can design seja comum ter técnicas de empatia que são trabalhadas de forma consciente, na arquitetura isso tem sido feito de forma mais subconsciente. Por isso, a exploração do usuário foi implicitamente integrada e, portanto, diluída em uma investigação inicial mais

ampla, vinculada à garantia da funcionalidade do programa. A obra de Richard Neutra apresenta-se como adequada para a realização deste estudo, devido ao foco constante que o arquiteto coloca na busca do bem-estar das pessoas através dos espaços habitados que projeta. O texto está estruturado em três partes diferenciadas: atenção às dimensões fisiológicas e psicológicas da pessoa; a análise da relação que se estabelece entre a pessoa e a natureza por meio de sua arquitetura; e a apresentação de exemplos específicos de técnicas aplicadas pelo arquiteto para conhecer o usuário, sua estrutura familiar e suas necessidades. Através dos casos estudados e com o apoio do feedback específico de alguns dos seus clientes, confirma-se a forte orientação para o utilizador na arquitetura da Neutra, procurando garantir o seu bem-estar físico e emocional através da configuração dos espaços, bem como da ampliação de ser através da conexão com a natureza. Um arquiteto à frente de seu tempo, que constrói um forte vínculo empático com seus usuários e aplica uma série de técnicas de empatia que terão um longo desenvolvimento posterior dentro do que hoje é conhecido como Design Thinking. (a) empatia, arquitetura centrada no usuário, processo de design, humanismo, Case Study Houses, Casa Miller, Casa Bailey, Eames 
Diseñar espacios arquitectónicos implica tener en consideración a los usuarios o habitantes futuros de los mismos. Es habitual leer descripciones detalladas de las estrategias de diseño utilizadas para atender $y$ satisfacer las necesidades de las personas Sin embargo, existen pocas referencias sobre el proceso de empatía que se ha llevado a cabo de los métodos, herramientas y procesos que se han utilizado para empatizar con dicho usuario con que sejetivo de conocer sus necesidades físicas, emocio con o sociales relacionadas con la futura obra arquitectónica.

Charles Eames compara el papel del diseñador con el de un buen anfitrión que anticipa las necesidades de sus invitados: "independientemente de que se refiera a diseñar una silla, planificar una exposición o recibir visitas para cenar todo gira en torno a anticipar las necesidades de sus invitados" (Cabrero-Olmos, 2027). El uso de técnicas de empatía como procedimiento consciente está quizá más extendido en el campo del diseño. En arquitectura este trabajo de conocimiento del usuario se ha venido haciendo de una manera más subconsciente, implícito en una investigación más amplia vinculada a garantizar la funcionalidad del programa, y por lo general, ha quedado oculto por la comunicación de los resultados de la obra arquitectónica.

A pesar de que no se nombra de forma explícita como una fase de "empatía", podemos observar en la historia de la arquitectura más reciente algunos arquitectos que prestaban especial atención al usuario. Richard Neutra (1892-1979), arquitecto austro-americano conocido por introducir el estilo internacional en la escena arquitectónica americana del sur de California, es un buen ejemplo de ello.

El usuario cobra especial relevancia Richard Neutra cuando se trata de configurar espacios y relacionarlos con el entorno. Lo que convertía a Neutra en un arquitecto único no era la búsqueda interminable de la forma, sino la interminable búsqueda de la esencia humana (Lamprecht,
2004). Su trabajo muestra un interés profundo en el cuerpo, sus hábitos y hábitats, buscando transformar la ecología humana (Lavin, 1999). Estaba convencido de que extraordinario, la tónica que marcaría su época estaría dada por el desarrollo de las ciencias biológicas (Neutra 1973, p.11), constituyendo este 'nuevo humanismo' el fundamento de la arquitectura'

Debido a la atención que el arquitecto demuestra hacia el conocimiento de dicho usuario y la satisfacción de sus necesidades vitales a través del espacio arquitectónico diseñado, su obra resulta de interés para realizar una aproximación desde el punto de vista del usuario y/o

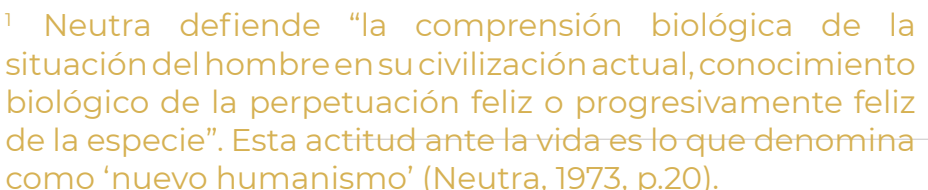
situación del hombreensucivilizaciónactual, conocimiento como 'nuevo humanismo' (Neutra, 1973, p.20). cliente $^{2}$. Adecuar un objeto al consumidor, significa, en primer lugar, conocer al consumidor (Neutra, 1973, p.20). Es precisamente este pensamiento de Richard Neutra el que da pie al desarrollo de la presente investigación más pormenorizada de mecanismos y técnicas que el arquitecto utiliza para detectar las necesidades del usuario y algunas de las estrategias arquitectónicas que despliega para satisfacerlas a través de sus diseños.

En este sentido, el artículo se estructura en tres partes diferenciadas. En primer lugar, se estudia la atención hacia las dimensiones fisiológica y psicológica de la persona. Se continúa con un análisis de la relación que se establece entre la persona y la naturaleza a través de su arquitectura. Y por último, se presentan ejemplos específicos de técnicas aplicadas por el arquitecto para conocer al usuario, su estructura familiar y sus necesidades. que realiza el encargo y 'usuario' es la persona que disfruta del espacio arquitectónico. En los textos que se har habitual encontrarse el término 'cliente'. Si bien, muchas entendemos en esta investigación. 
Atención a las dimensiones fisiológica y psicológica de la persona

Búsqueda del bienestar físico y psicológico del usuario.

La motivación que lleva al arquitecto a indagar La molivaionte subre la esencia humana eragu continuamente sobre la esencia humana era su convencimiento, bien sencillo, de que la buena arquitectura sana, mividus se ve influenciada de manera constante por el ambiente cotidiano y los seres humanos que están cerca y presentes durante periodos de tiempo prolongados; es decir, por el hogar y la familia (Neutra, 1972, p.70).

Desde una perspectiva arquitectónica, esto significa que todas aquellas decisiones tomadas durante el provecto y llevadas a cabo en la obra final, tienen influencia en el bienestar del usuario. Independientemente de que se cobre conciencia de ello o que el asunto pase inadvertido, respondemos a nivel biológico a cada una de las experiencias espacio-temporales que vivimos. "Nuestro espacio es siempre la interacción de nuestra dote orgánica y de las sugestivas circunstancias que nos rodean" (Neutra, 1972, p.229).

Se puede decir, entonces, que la experiencia diaria del ser humano puede calibrarse por su relación con el entorno, y aquí juega un papel fundamental la tarea del arquitecto en cualquier marco

\section{$\underline{ }$} 3 El arquitecto entiende que una 'buena arquitectura' mientras que una 'mala arquitectura' aliena lo humano de
la naturaleza y de su naturalidad esencial. 4 A través de la metáfora de que "el menudo y permanenpone de relieve la necesidad de prestar atención a todos
Tener en cuenta las necesidades ambientales y fisiológicas de la persona es de vital importancia para la tarea que se desarrolla. En uno de sus escritos, el arquitecto expresa que "en la sala de un tribunal, la justicia está condicionada por los cambios de calidad del aire, por el soporífero ácido butínico y por los olores corporales"s. Sin embargo, Neutra va más allá de las necesidades puramente fisiológicas del usuario, tratando de unificarlas con el bienestar psicológico.

El científico en Neutra calculaba las necesidades de aire en un cuarto de baño, pero al artista le sacaba de sus casillas que objetos 'modestos', como un portapapel de no estuvieran diseñados con sentido estético, siguiendo las teorías de la Gestalt y dejaran de ejercer un efecto positivo sobre el ojo y el cerebro y, por consiguiente, sobre el bienestar del observador (Lamprecht, 2004, p7-8)

El arquitecto defiende que no hay una división entre lo 'utilitario' y lo 'estético', que esta dualidad no existe en la naturaleza exterior, compuesta de elementos integrados en su totalidad.

\section{Biorrealismo. Sustrato corporal de la vida mental.}

Esta unión entre lo fisiológico y lo psicológico caracteriza su modo de afrontar la arquitectura y la vida en general. Define su filosofía como 'biorrealismo' y pensaba que la arquitectura debe dirigirse por el modo en que los seres humanos se comportan y evolucionan.

Se ve influenciado por la lectura de los escritos de Wilhelm
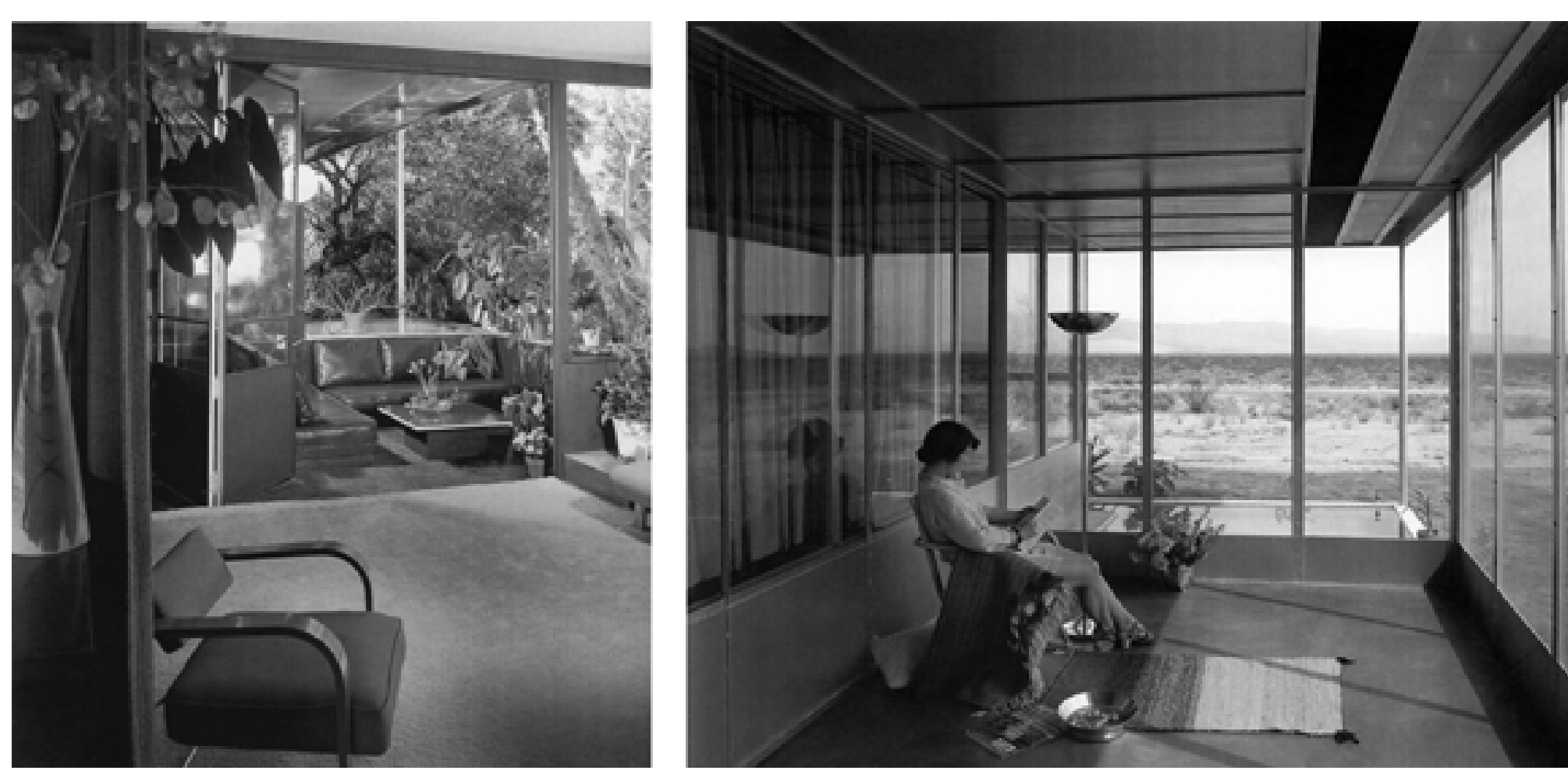

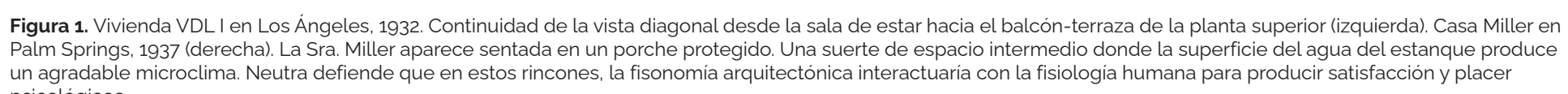
un agradable
psicologicos 5 Escrito no publicado, del 14-02-1969: "Sozial psychologie
und Architektur", traducción al inglés de Alphonz Lampre-
cht, Archivo de Neutra, Universidad Politécnica del Estado
de California. Pomona. (Lamprecht, 2004 . 7 ). de California, Pomona. (Lamprecht, 2004, p.7) 
Wundt ${ }^{6}$ durante su etapa de estudiante, $y$ en concreto, por el libro Principios de psicología fisiológica (1874), cuyas investigaciones sentaron las bases para la medición de la sensación física. El título del casítulo de introducción de dicho libro "sustrato corporal de la vida mental", parece definir la visión del arquitecto de una nueva arquitectura en sintoní con las necesidades bióógicas (lo que podrí considerarse como búsqueda de un nuevo tipo de vida).

Desde su concepto de biorrealismo, Neutra concibe el buen diseño como una medicina para diseño como una medicina para el bienestar fisico. Incluso llega a plantearse la diciones que conducían a la miserio al hambre, como menciona en el proyecto de los Centros Sociales en Puerto Rico (1944-45).

Defiende una arquitectura que sane al individuo, y precisamente una de sus primeras obras y de las más conocidas, la casa Lovell (1927-29), ha sido bautizada habitualmente como 'la casa de la salud".

Philip Lovell propagaba sus teorías sobre la salud y la higiene por medios naturales. A grandes rasgos, su teoría consistía en que se aseguraba mejor el bienestar físico y la felicidad de las personas a través de un buen alojamiento que mediante el uso de medicamentos y drogas.

Arquitecto y cliente compartían el mismo punto de vista por lo que "el cliente ideal había encontrado su arquitecto ideal". (Sack, 1994, p.14).
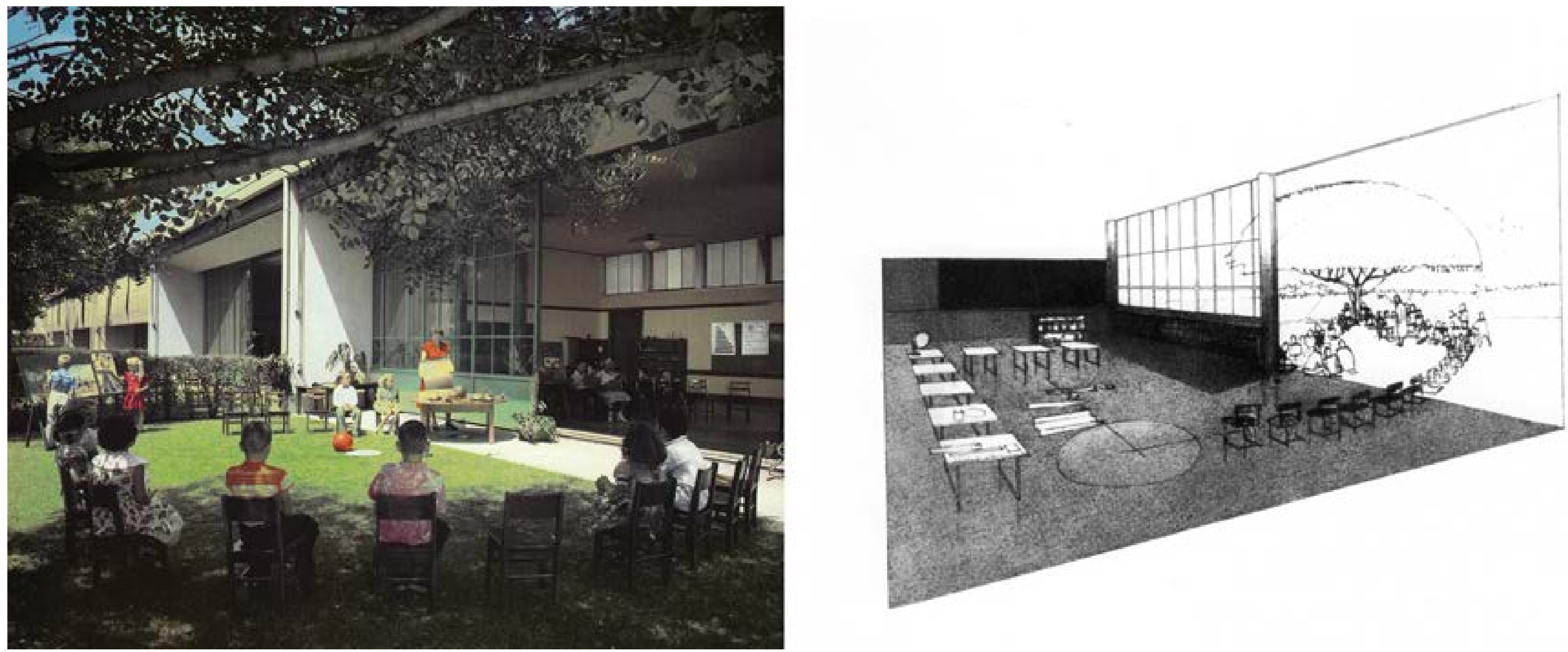

Figura 2. Colegio Corona en Los Ángeles, 1935 (izquierda) y Colegio Emerson en Westwood, 1938 (derecha). A través de la comunicación de ambos proyectos, fotografía 
La aplicación de los principios de la teoría de percepción de la Gestalt, conocidos desde su estancia en la Bauhaus, le permitieron atender a la dimensión más psicológica de la persona a través de las decisiones de diseño. La búsqued de la belleza, un sentido de equilibrio y la creación de un efecto positivo en el ojo y en el cerebro son constantes en sus obras arquitectónicas.

Neutra siempre se preocupó por lograr una unidad entre la casa, sus muebles y sus habitantes, sin establecer diferencias entrelasviviendas debajocostey sin establecer de lujo?.

La relación con la naturaleza

\section{La naturaleza somos nosotros mismos}

La naturaleza tiene especial relevancia y presencia en la obra del arquitecto, que viene derivada de su firme creencia de que la naturaleza somos nosotros mismos.

El universo del que formamos parte es un continuo dinámico. Se extiende desde los sistemas galácticos más distantes hasta nuestra atmósfera, nuestra biosfera y nuestro manto terrestre, penetrando cada vez más profundamente en la red de acontecimientos moleculares y subatómicos que configuran toda la materia, el movimiento y nuestra mente. Nuestra pie es una membrana, no una barricada... Los límites más

7 En su viaje de 1930 a Japón, Neutra observó caracterís ticas similares en las casas para los ricos y para los pobres dependiente de poder adguisitivo perfeccionada con tecnología del siglo XX (Lamprecht, 2004). remotos del cosmos no se encuentran 'ahí fuera, en con los pli', gino qús están causalmente entrelazados paisaje interior ${ }^{8}$.

En sus escritos, muestra la intención de realizar estudios sobre sobre el modo en que la naturaleza exterior se relaciona con la naturaleza íntima del hombre; estudios de lo que de los seres humanos reconocidos y gratificados, no durante un momento sino durante toda la vida de su grupo (Neutra, 1972 p.266). Tomar en serio al individuo grupo (Neutra, 1972, p.266). Tomar en serio al individuo allá de las modas pasajeras. En consecuencia, en la arquitectura de Richard
Neutra se puede hablar de una continuidad entre el ser humano y la naturaleza del entorno. Una acción ser humano y la naturaleza del entorno. Una acción proyectual derivada de esta ausencia de division entre la interrelación entre naturaleza como medio y naturaleza propia de los seres humanos.

\section{Continuidad entre interior y exterior}

Muchos son los ejemplos que permiten ver las estrategias que utiliza el arquitecto para fundir el interior y el exterior en sus obras de arquitectura, hasta el punto de estar "sentado en un salón de Neutra y tener la sensación de encontrarse fuera de la casa".

- Cita recogida en los ensayos póstumos Nature Near: 9 Percepción de Arthur Drexler, director del departamen-
to de diseño en el MoMA, recogida en el libro "The Archi-
tecture of Richard Neutra" editado por el museo con motivo de la exposición realizada sobre el arquitecto.
La terraza separada del espacio interior mediante un tabique corredero de vidrio es un recurso habitual. Se da continuidad a los acabados de techo y suelo, y dicha terraza solía disponer de calefacción en el suelo para dotar de más ambigüedad a los límites entre espacio interior y exterior. En sus primeras obras emplea ventanas altas con alféizares bajos, aunque progresivamente realiza una transición hacia los muros de vidrio de suelo a techo.

En las viviendas genera unos espacios intermedios o corners que transmiten una sensación de expansión del ser interior (Figura 1). A nivel perceptual, alargan os espacios a través de vistas diagonales y permitir un alcance más amplio para la visión periférica. En palabras de Sylvia Lavin, es "el rasgo mas célebre de la arquitectura de Neutra, donde se acumulan inestabilidades e incertidumbres y donde se proyectan los deseos, tanto psiquicos como orgánicos" (Lavin, 1999, p.22).
Esta idea de continuidad estaba presente en todos sus edificios, no solo en las viviendas. Así, en el diseño de colegios, se puede ver cómo fundía el espacio de
aprendizaje incorporando al aula convencional los beneficios del espacio natural exterior (Figura 2).

\section{Examinar los solares}

Elegir el lugar para la vivienda así como la disposición y orientación de ésta en la parcela es una cuestión clave para el arquitecto. "Los seres humanos no habitan un espacio desprovisto de orientación, tal y como lo conciben abstractamente el agrimensor o el geómetra. Por el contrario, la orientación, una dirección dada, es cosa innata en todos" (Neutra, 1972, p.231). En este sentido, examinar los posibles solares para sus clientes $\mathrm{y}$ ayudarles a elegir qué parcela escoger para construir su futura vivienda es una de las primeras acciones Ilevadas a cabo por el arquitecto. Hay varias referencias que dan muestra de ello. 
Cuando proyectó la casa para Alan y Janet Glen en 60), pautó con los clientes una visita a los solares seleccionados un día de luna llena. Quería visitar el terreno en unas condiciones que permitieran decidir la mejor ubicación de lavivienda, considerando la experiencia tanto diurna como nocturna. Además, pasó con ellos dos semanas completas, lo que le permitió conocer de primera mano su forma de vida, sus intereses y necesidades. En el caso de la vivienda para Grace Miller en Palm
Springs (1937), la historia de la familia narra que Neutra llegó en un automóvil enganchado a un remolque en el que transportaba una mesa giratoria de dibujo y un toldo. Mientras dibujaba sentado a la sombra el dispositivo de giro le permitió estudiar los ángulos del sol y del viento (Lamprecht, 2004, p.43).

Conocimiento del usuario y de la estructura familiar.

Empatía y relación de confianza

Richard Neutra defendía la necesidad de construir una relación de confianza con el cliente, similar, siguiendo la metáfora que él empleaba, a la que un médico construye con sus pacientes. Si no hay empatía $a^{10}$, el arquitecto a lo sumo podrá ofrecer a sus clientes una obra que habrá nacido muerta, por mucho que la haya elaborado con lógica y cuidado, y aunque su construcción se ajuste a principios consecuentes. Asi, escribia:
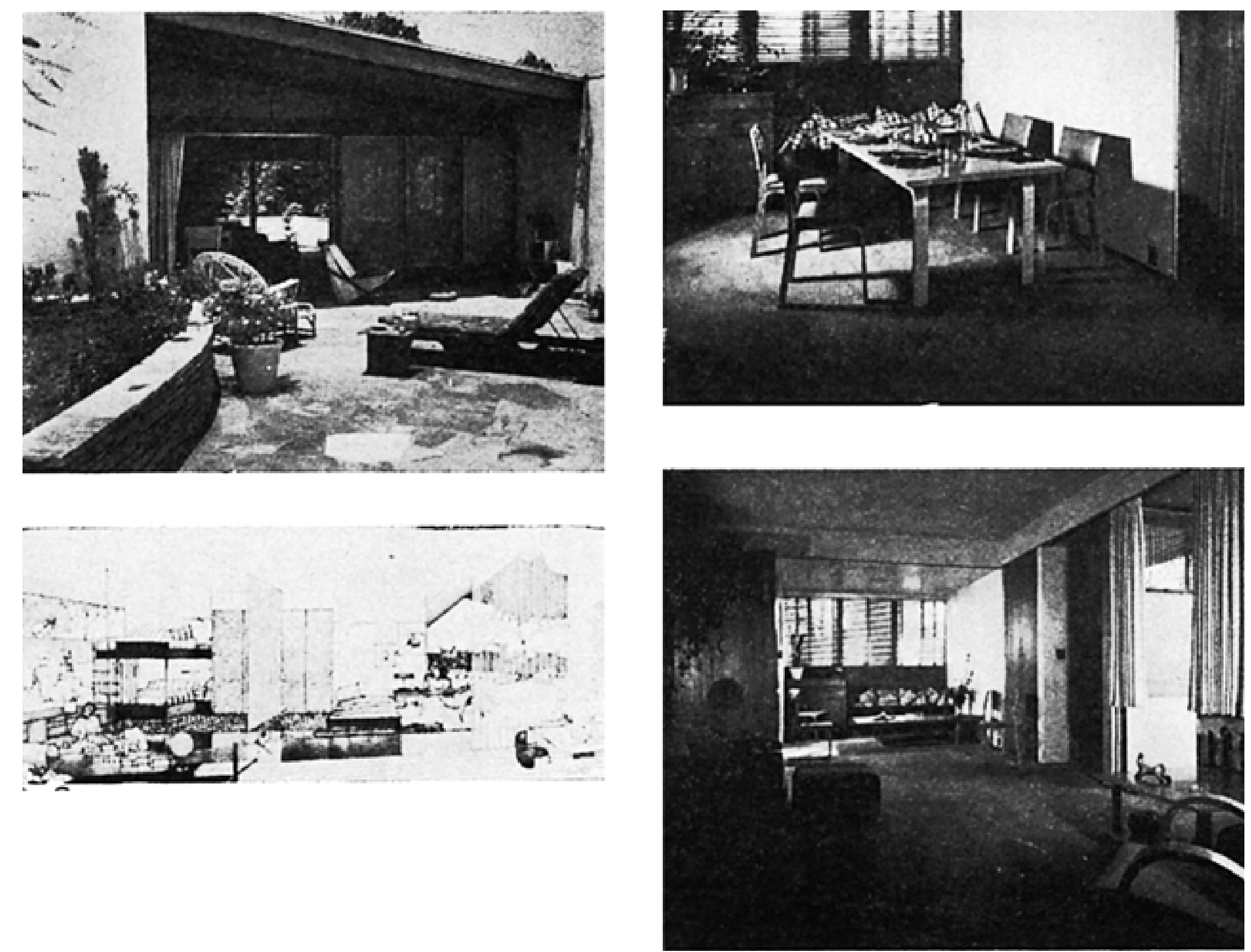

Figura 3. Selección de fotografias e ilustraciones del álbum de recortes aportado Mr. Omega en un momento de la entrevista. Mr. Omega, através de estos ejemplos, pone
el foco en la relación entre zona de estar y patio (a), en una habitación equipada para niños (b). en una mesa de doble posición (alta para comery baja para tomare el teé) que ell foco en la relación entre zona de estary y patio (a), en una habitación equipada para niños (b), en
permite adaptar el uso del espacio (c) y en la comodidad de andar descalzo sobre suelo radiante (d)

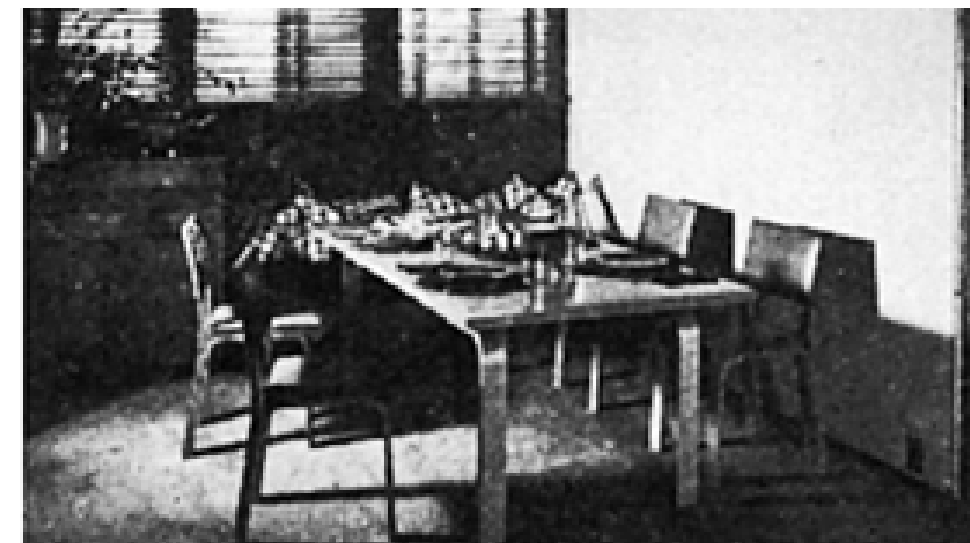


Ninguna obra de éxito puede nacer sin un factor humano, sin esa chispa humana incalculablemente valiosa que se (N participa activamente an

Giedion añade que ese excelente contacto entre arquitecto y cliente resulta aún más notable por el hecho de que economí sucurinstintos equivocados (Boesiger (ed) 1950).

Su apasionado y directo compromiso con sus clientes, y todavía más con los ocupantes de sus casas, le condujo menudo a desistir de sus proyectos alemanes (Sack, 1994, p.21).

Observación e intuición

Neutra pone de relevancia dos métodos de investigación el más racional, analítico y metódico, frente al emociona e intuitivo. Para poner en valor este segundo método, se vale de una experiencia médica vivida con su hermano enfermo, en la que el diagnóstico no vino de un análisis científico de laboratorio sino de la observación e intuición del médico experimentado. Sin descartar la necesidad de una investigación metódica y detallada en el proceso de proyecto (que le parece imperativa) defiende también que "un clínico dotado de intuición es a menudo la persona más adecuada para orientar y ofrecer líneas de experimentación al investigador" (Neutra, 1972, p.63).

La observación es uno de los métodos de empatía que permite captar informacion sobre los comportamientos y necesidades de los usuarios. Así, en referencia al proyecto de la casa Lovell, se lee:

Antes de empezar el proyecto, Neutra se había dedicado a observar a la pareja y a sus tres hijos durante largo tiempo; estudió sus hábitos de comer, beber y dormir, observó sus momentos de ocio, escucho sus ideas e ilusiones, buscó conexiones fuera de la familia, amigos, diversiones; para el proyecto de la cocina, interrogó a la familia y al cocinero (Sack, 1994, p.14).

Entrevista

El arquitecto reconoce que las particularidades de sus clientes siempre fueron para él una fuente de inspiración y menciona el contacto activo con ellos como su princión fuente de conocimiento.

Durante las entrevistas y conversaciones con los usuarios, hay que estar preparado para comprender y alvia tensiones y fricciones interminables (habituales entre los miembros de la unidad familiar), procurando no irritarse miembros de la unidad familiar), procurando no irritarse con los clientes. Senala que la expresión puramente verbal la necesidad de atender a la comunicación no verbal".

Defiende el uso de un lenguaje sencillo en la comunicación con los usuarios. "La gente siempre tiene derecho a que se le hable en un lenguaje que entiende" (Neutra, 1972, p.90) por lo que observa la capacidad del cliente para interpretar

A través de la observación activa se puede captar infor macion adicional y nutritiva no comunicada verbalmente nificativos y duraderos rasgos del rostro, y cuando éste se de fatiga, temor o entusiasmo. La actitud del individuo que to de los dedos, las manosy es una silla, clerto movimienmedad que agrega brillo a los ojos, la aclaración vacilante de la garganta, todas las formas de expresión de la conduc tá, iluminan una conversación atenta si uno está dispuesto a ver yrabian cabo en 1967, estableció que solo el $7 \%$ de la transmisión emocional de la comunicación se atribuye a las palabras, el $38 \%$ al lenguaje paraverbal (tono, pausas, entonación faciales, gestuales y posturales.

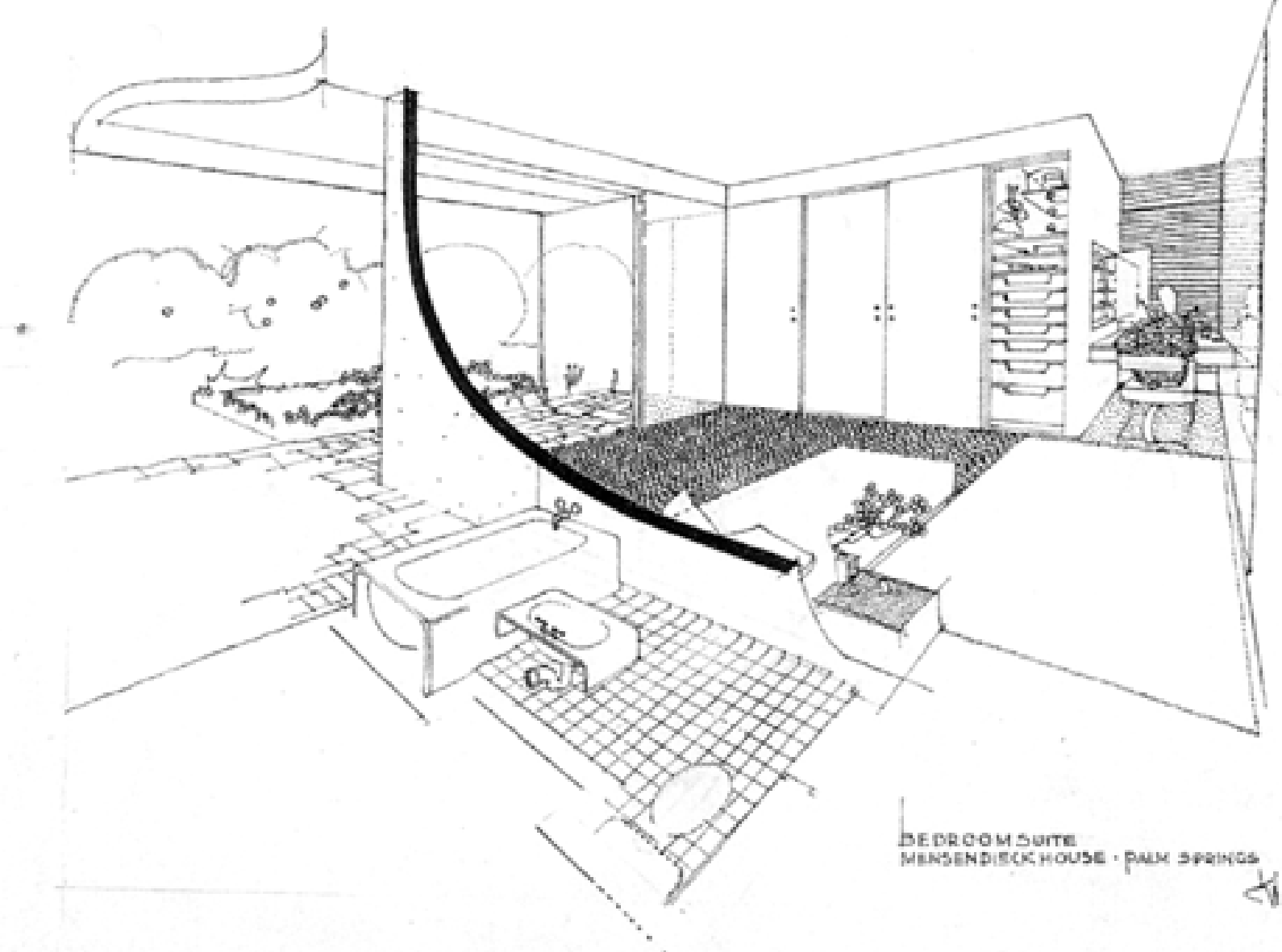

Figura 4. La ilustración muestra el dormitorio y el baño de la casa para la Sra. Miller, destacando el diseño de la mesa de aseo con su plancha de ónice y el armario ropero 
$\Leftrightarrow$

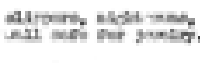

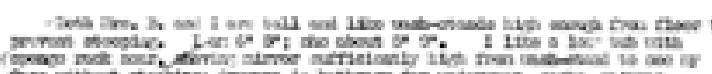

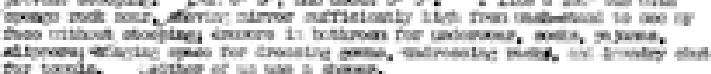

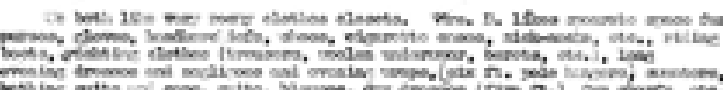
con ratis Ain han

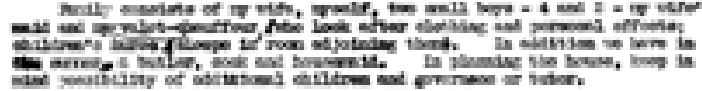

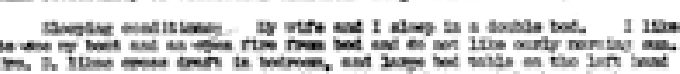

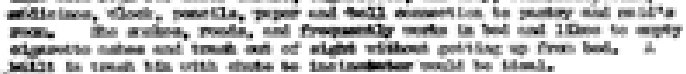

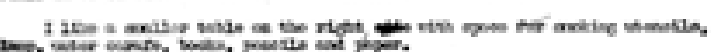

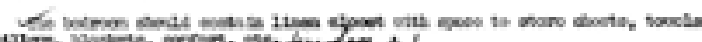
num

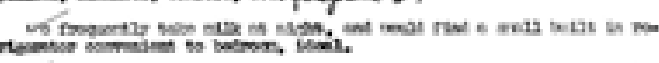

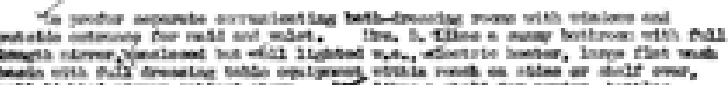

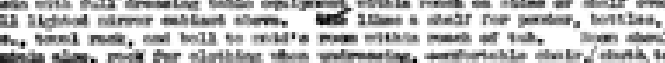

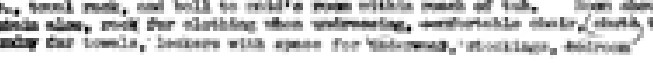

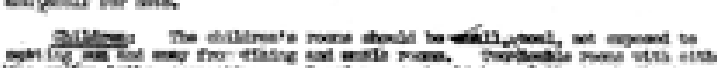

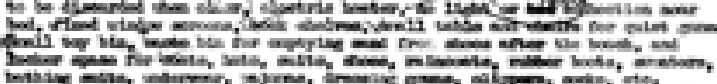

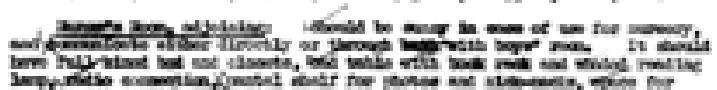

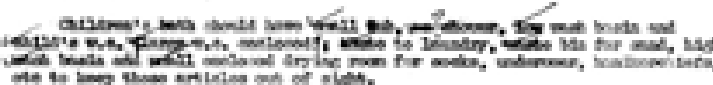
-

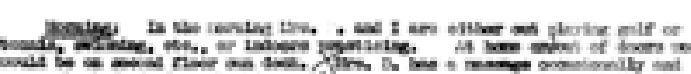

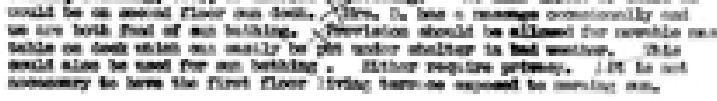

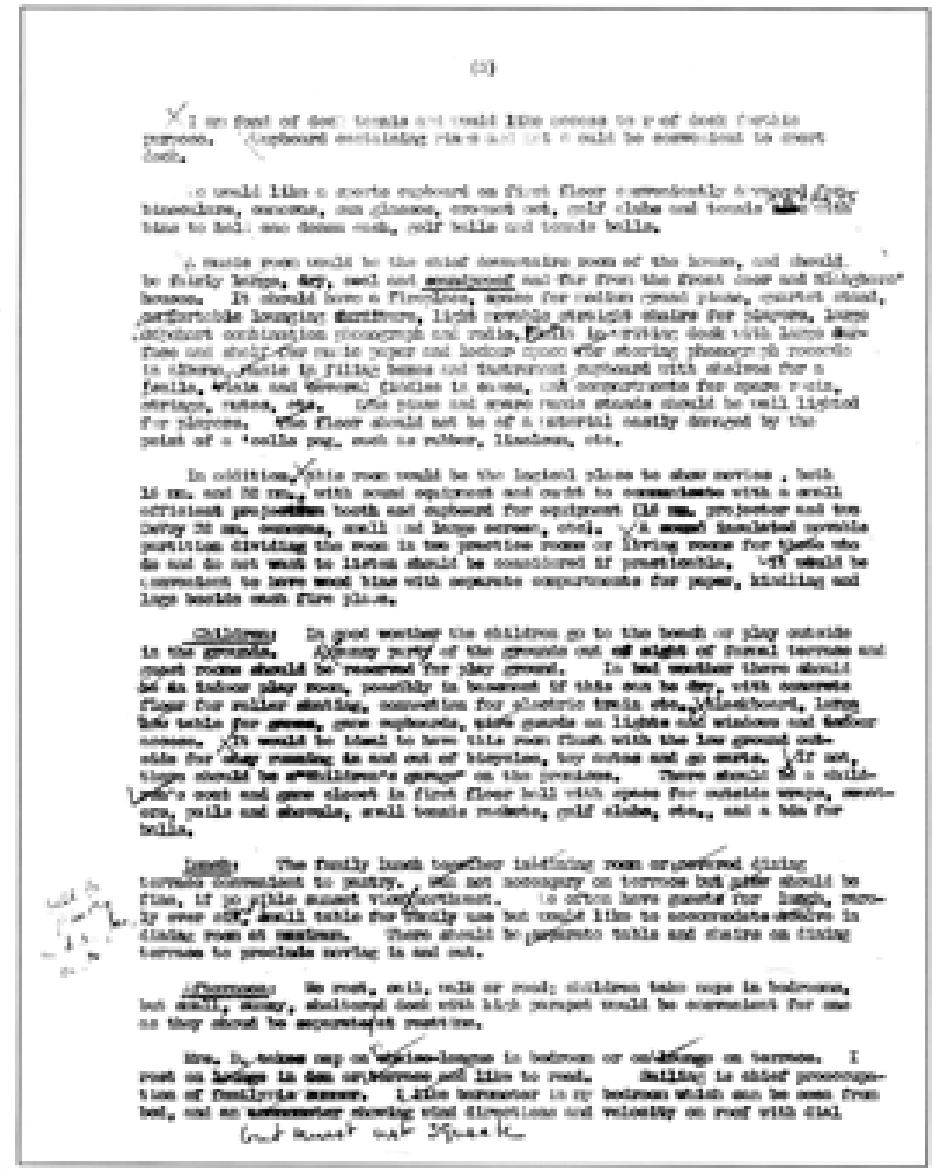

Figura 5. Facsimil del memorándum que la familia Brown envió al estudio de Richard Neutra en contestación a su cuestionario de cliente. Se muestra un extracto de las planos y asimilar ideán

Cuestionario de cliente

Como complemento a estas observaciones directas y entrevistas verbales con los usuarios, en el estudio preparado para conocer al usuario en profundidad y detectar cuestiones clave para el proyecto. Se realizaban preguntas detalladas sobre sus deseos, sus formas de vida y sus antecedentes Se pedía a los usuarios que entregasen respuestas por separado en la medida de lo posible y que las acompañaran de imágenes informales de ellos mismos o de mobiliario que esperaban usar (figura 3) (Lamprecht, 2019, p.42).

En ocasiones, las preguntas podían ser tan detalladas como en el caso de la Sra. Miller. Saber su número de jerséis o de sombreros, entre otros, permitieron diseñar los elementos arquitectónicos y de mobiliario que respondían directamente a las necesidades de la usuaria (figura 4).

Este cuestionario constaba de dos columnas, una titulada 'necesidades del cliente' y otra 'solución arquitectónica', en la que se anotaba si dicha respuesta debía plasmarse en la planta, la sección o el alzado. En la figura 5 se ve un extracto del memorándum de siete páginas realizado por la familia Brown para responder a dicho cuestionario. Los apartados clave desarrollados en el escrito son: clima, vida diaria, profesión y localización de la parcela.

Construcción de perfiles de usuarios

Estas entrevistas, observaciones y cuestionarios derivan en la construcción de perfiles de usuario para sus viviendas. La primera constancia del empleo de esta tecnica de empatía por el arquitecto se encuentra en sus propuestas para el programa Case Study Houses (CSH), donde menciona que 
la CSH n`13 está diseñada para el Sr. y Sra. Alpha, mientras que la $\mathrm{CSH} n^{\circ} 6$ se diseña para el Sr.y Sra. Omega (figura 6).

Es curioso que en el marco de este mismo programa de viviendas promovido por la revista Arts \& Architecture surja otro caso claro de creación de perfiles de usuario. Charles y Ray Eames utilizan una representación gráfica de las siluetas de los futuros habitantes de las Case Study Houses $n^{\circ} 8$ (ellos mismos) y nْ9 (John Entenza) rodeadas por un conjunto de artefactos para definir sus respectivos estilos de vida. (Fernández-Villalobos, 2019). Denominación de espacios a través de su función

A la hora de definir el programa de las viviendas, el arquitecto evitaba los nombres convencionales como 'cocina' O 'salón' buscando denominar a los espacios por las funciones que albergaban. Este cambio en el lenguaje, eligiendo un verbo de acción, es aparentemente sencillo y sin embargo, abre un mundo de posibilidades e invita pensar características del espacio desde una perspectiva más amplia. Se evocan las necesidades del usuario para llevar a cabo esas acciones, evitando traer la primera imagen asociada a las habitaciones que conocemos.

La casa Miller (1937) es buen ejemplo para ilustrar este punto, ya que trataba las funciones de la misma manera que las transiciones espaciales'2. "Nuestras habitaciones no se llaman salón, comedor, dormitorio,... Las habitaciones son porciones del gran espacio en que vivimos y son pragmáticamente elásticas" (Lamprecht, 2004, p.43).

El espacio viene derivado de las necesidades directas de su usuaria, Grace Lewis Miller, que se dedicaba a enseñar el

12 Quizá se puede ver aquí la influencia de la casa Schröder, diseñada por Gerrit Rietveld en Utrecht (1924), donde su flexible diversificación de funciones dentro del mismo espacio (Lamprecht, 2004, p.9).

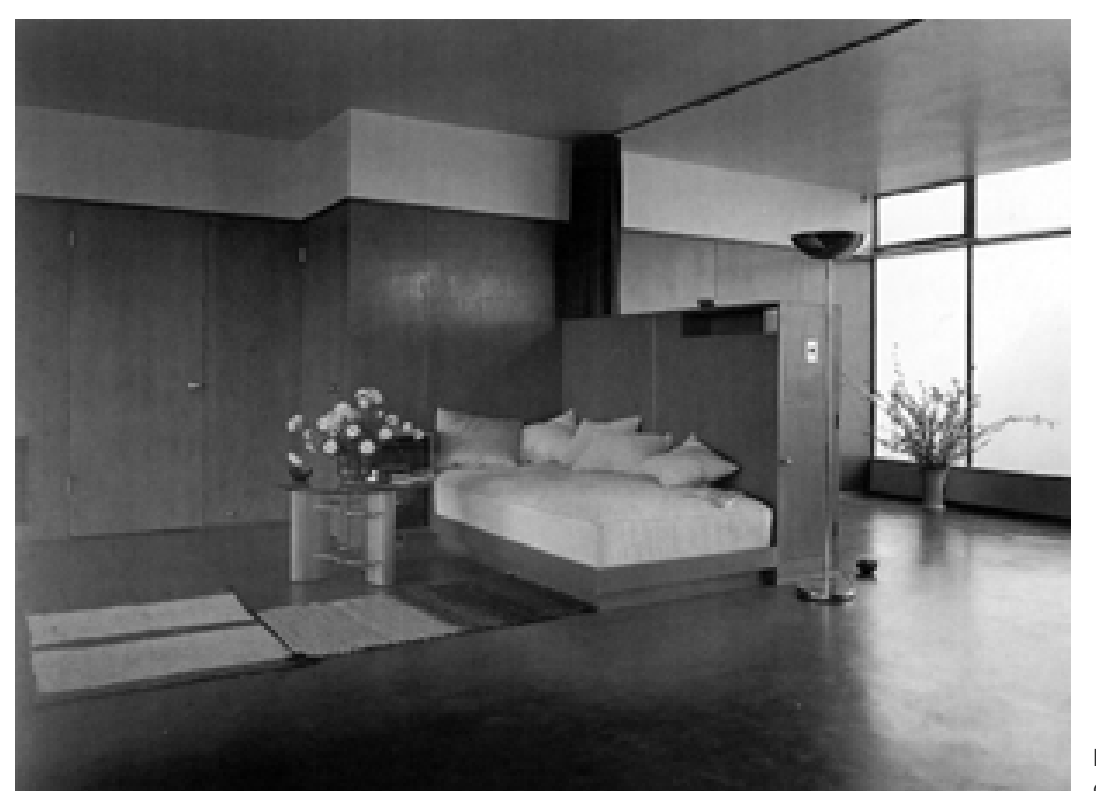

Figura 7. Espacio flexible principal de la casa Miller en Palm Springs, 1937. La cortina junto al camino protegia frente a miradas el estudio en la casa (al norte) y la sala

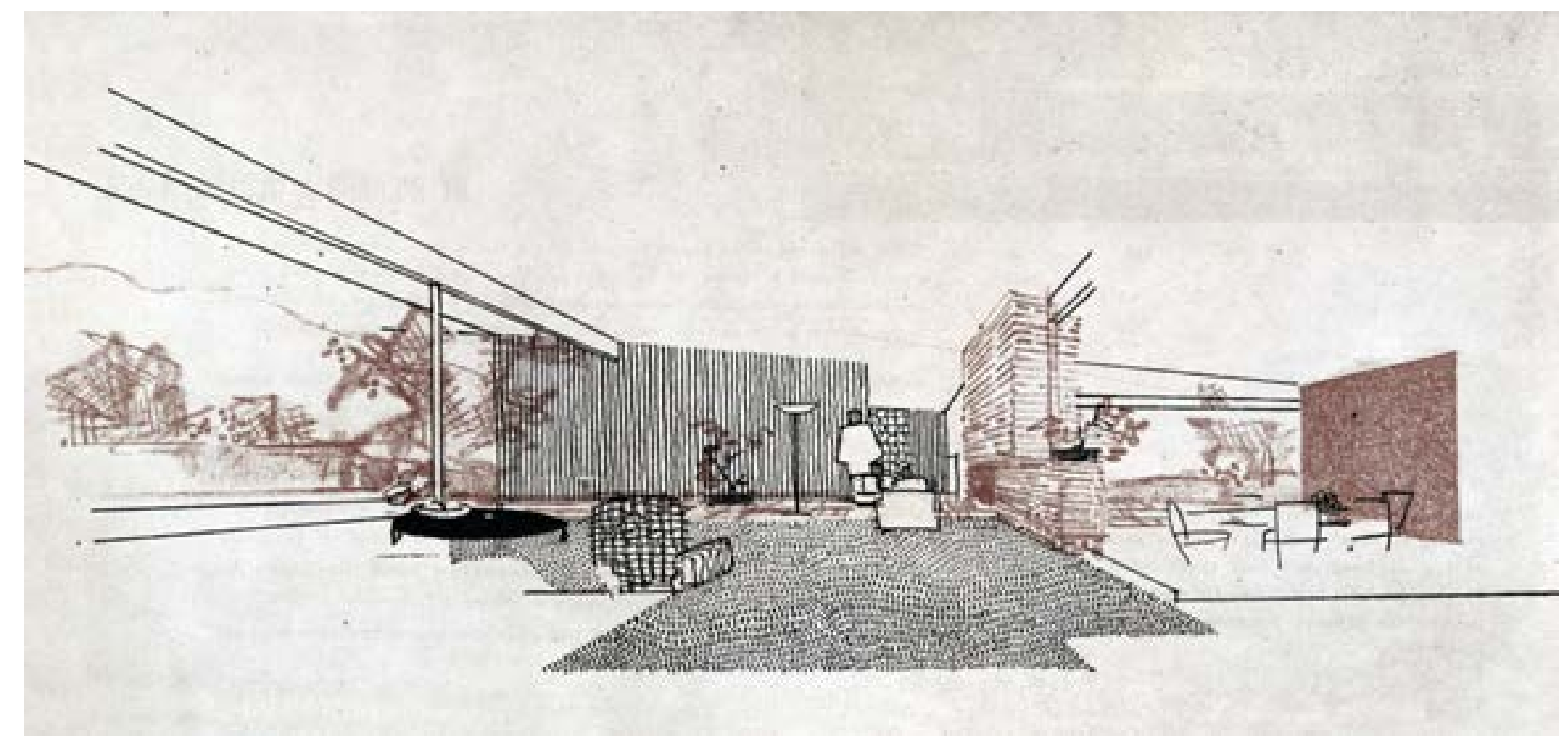

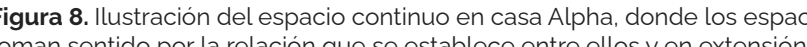
toman sentido por ler.
hacia la naturaleza. 
Aunque en la arquitectura de Neutra se detecte como constante la utilización de las plantas en forma de cruz como por ejemplo en las casas Kaufmann y Tremaine, la configuración de los espacios sociales es muy diferente configuracion de los espacios sociales es muy diferente diferentes y en ese conocimiento profundo del usuario que determina el curso posterior del proyecto.

La estructura en cuatro patios hace alusión a la dimensión social que toman los espacios. Cada uno de los patios tiene un cometido o uso principal y va asociado directamente con los espacios interiores con los que se relaciona. Tomamos el ejemplo de la $\mathrm{CSH} n^{\circ} 6$ para ilustrar este aspecto (figura 9), y vemos en la planta la referencia cuatro patios: Patio de entrada (C1), patio social (C2), patio deportivo o de ocio (C3) y patio práctico o de servicio (C4).

Resulta reseñable que al ser el usuario considerado parte fundamental del proceso de proyecto, tanto los dibujos de

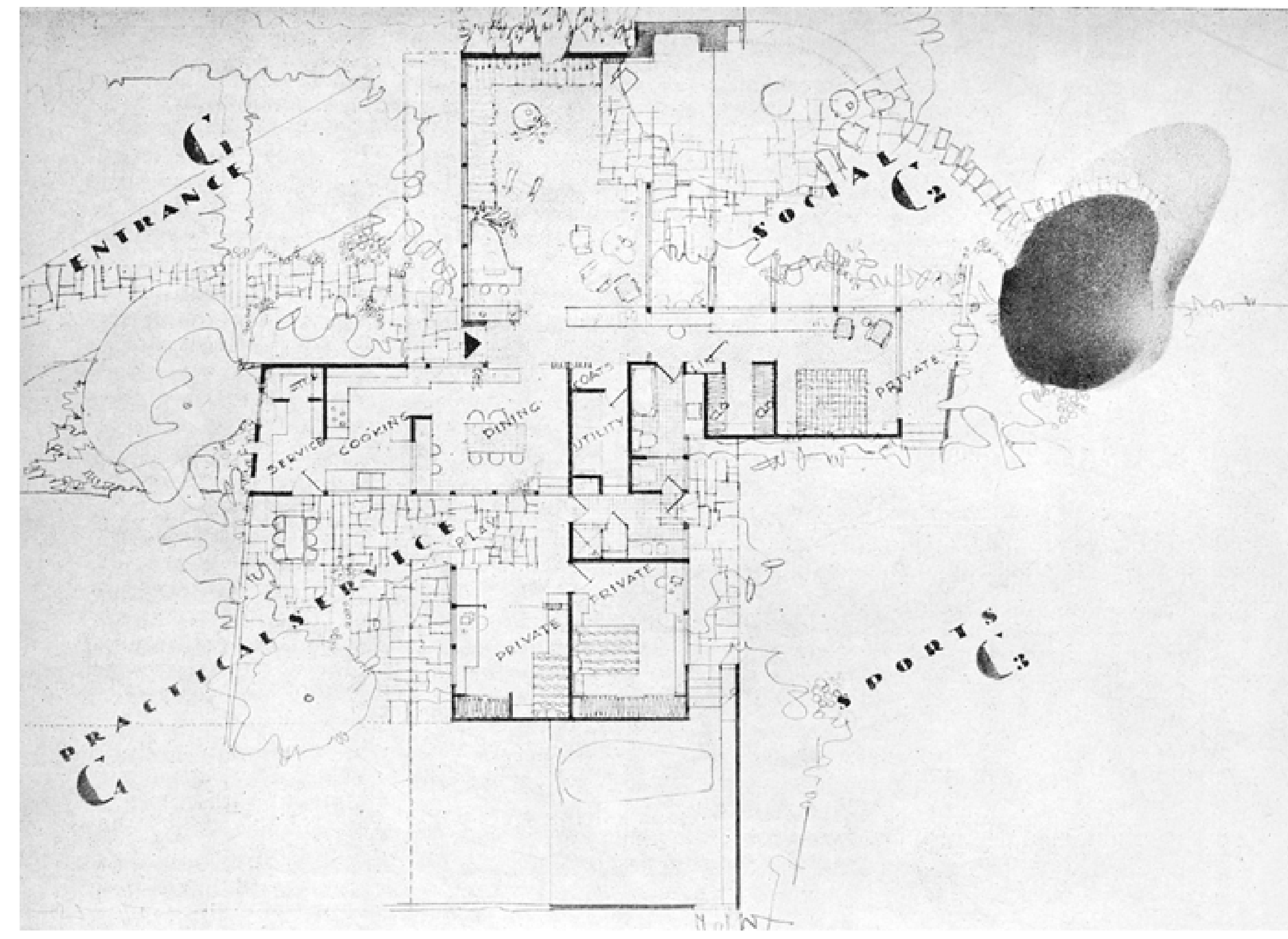



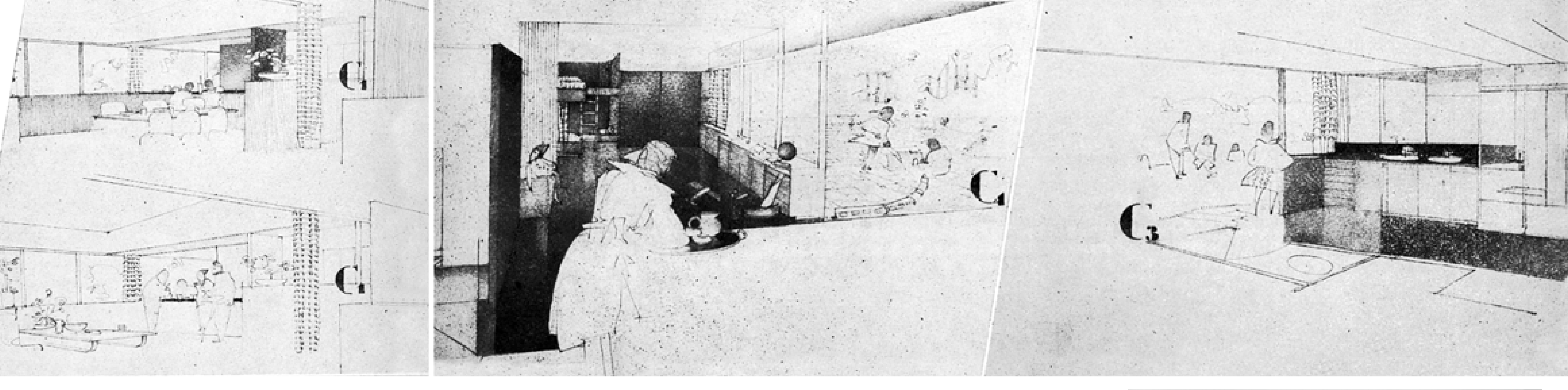

Figura 10. Plano de la casa Omega o CSH n॰6 donde se hace notar forma visible l
vinculàndolos directamente con los espacios interiores con los que se relacionan

las ideas como las imágenes de los espacios arquitectónicos se muestran siempre con el usuario en acción y llenos de vida (figura 10).

"estaba conceptualmente 40 años adelantado a su tiempo" (Lamprecht, 2019, p.42). A pesar de que estas teorías que combinan psicología y filosofía, biología y ecología no estaban científicamente demostradas, se ven apoyadas en su amplia experiencia práctica y producción arquitectónica, así como en la inquebrantable fe del arquitecto de "estar haciendo las cosas bien" (Sack, 1994, p.19).

Resulta interesante reseñar algunos de los mensajes que Neutra recibe de sus clientes y usuarios a modo de feedback. Muestran aspectos relevantes del uso y disfrute de los espacios ${ }^{1 / 4}$ y refuerzan esa sensación de 'hacer las cosas bien'. Veamos, a continuación, tres ejemplos:

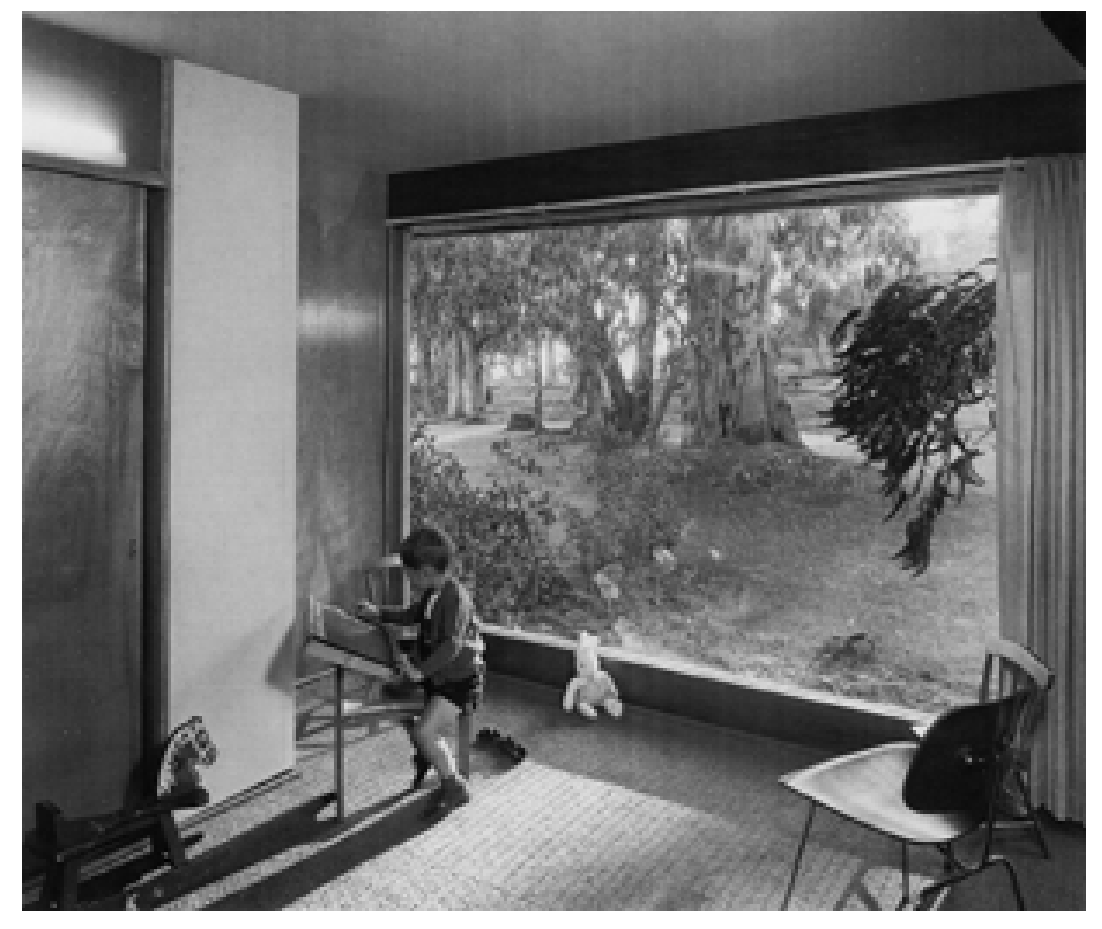


Charles y Ray Eames viven durante siete años en los apartamentos de Strathmore Drive en Los Ángeles diseñados por Neutra, donde comienzan su carrer profesional. En una carta enviada al arquitecto ponen de relevancia la riqueza que el espacio añade a sus vidas, pudiendo desarrollar su entorno de manera más expansiva, sintiendo que viven dentro de su propio jardín, manteniendo total privacidad

Melba Beard, a propósito de la casa Beard (1934-35), expresa: "Lamento que en nuestros últimos años no dispongamos de un lugar como nuestra casa de Altadena; proporcionaba una libertad de espíritu de proximidad a la naturaleza, una vida reposada, una como Neutra deseaba".

Y el Dr. Bailey afirma señalando los árboles situados más allá de las paredes de vidrio: "Lo que me qusta de esta casa es que no hay casa", en referencia a House $n^{\circ} 20$ donde habitaba (figura 11).

En conclusión, estos mensajes de feedback y los ejemplos estudiados a lo largo de la investigación confirman el éxito de la arquitectura de Richard Neutra como espacio que confiere bienestar físico y emocional a sus habitantes. Sus principios humanistas y la búsqueda de la esencia human a través de la práctica del biorrealismo, le llevan a situar a usuario en un punto central de su práctica arquitectónica.

Se demuestra que es un arquitecto con una fuerte orientación hacia las personas, que buscaba la expansión de su ser interior mediante la conexión del espacio construido con la naturaleza. Consideraba fundamenta la interacción directa con sus clientes para aportar valor los espacios que van a ser habitados, observando hábitos y modos de comportamiento que se verían reflejados en sus diseños.
Se trata de un arquitecto adelantado a su tiempo, que aplicaba de forma intuitiva métodos y procesos que

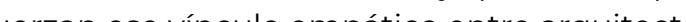
y usuario en el proyecto arquitectónico. Una serie de usuario en el proyecto arquitónico. Una serie de explícita tienen un largo recorrido posterior y sientan las bases de técnicas que hoy se manej dentro del pensamiento de diseño.

Disponer de medios y técnicas para conocer al usuario durante el proceso de proyecto es imprescindible para generar espacios arquitectónicos adecuados, tanto en su dimensión funcional como en la psicológica y emocional. A través de todos los sentidos nos penetran íntimamente impactos vitales que nos agitan e inquietan, por lo que, en palabras del propio Neutra, "el arquitecto del mañan en palabras del propio Neutra, "el arquitecto del manana humanidad, en medio del glorioso progreso de nuestra época". (Neutra, 1973, p.24).
Boesiger, W. (ed.). (1950). Richard Neutra Buildings and Projects. Zurich: Girsberger Verlag.

Cabrero-OImos, R. (2027). "Cuaderno de empatía: una buena práctica para conocer al usuario desde el inicio del proyecto" en JIDA'27. Jornadas de Innovación Docente en Arquitectura. Barcelona:
UPC. https://doi.org/10.5827/jida.2021.10598

Drexler, A.y Hines, T.S. (1984). The Architecture of Richard Neutra: From International Style to California Modern New York: The Museum of Modern Art.

Eames, C., Eames, R.; Ostroff, D. (ed.). (2015). An Eames Anthology. New Haven: Yale University Press.

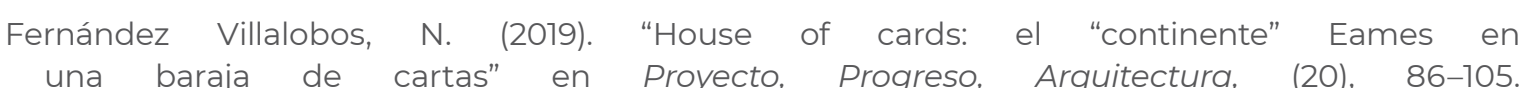
https://doi.org/10.12795/ppa.2019.120.05

Hines. T. (2005). Richard Neutra and the Search for Modern Architecture. New York: Rizzoli. (1ªed: 1982).

Lamprecht, B. (2004). Richard Neutra. La conformación del entorno. Köln: Taschen.

Lamprecht, B. (2019). Richard Neutra. Complete Works. Köln: Taschen

avin, S. (1999). "Open the Box: Richard Neutra and the Psychology of the Domestic Environment" en Assemblage, (40), 6, pp. 6-25. doi:10.2307/3171369

Neumann, D. (ed.). (2001). Richard Neutra's Windshield House. Mass: Yale University Press and Harvard Design Schoo

Neutra, R. (1957). Planificar para sobrevivir. Mexico: Fondo de Cultura Económica.

Neutra, R. (1972). Vida y forma. Buenos Aires: Marymar (1ªed. 1962).

Neutra, R. (1973). Realismo biológico: un nuevo renacimiento humanístico en arquitectura. Buenos Aires: Nueva Visión.

Ostwald, M.J., y Dawes, M.J. (2018). "Richard Neutra: Spatial Theory and Practice" en Mathematics and the Built Environment, 179-205. doi:10.1007/978-3-319-71647-3_6

Sack, M. (1994). Richard Neutra. Barcelona: Gustavo Gili. 
Smith, E.A.T. (2009). Case Study Houses. The Complete CSH Program (1945-1966). Köln: Taschen Travers, D.F. (2014). Arts \& Architecture 1945-49. Colonia: Taschen.

Travers, D.F. (2016). Arts \& Architecture. Archivo online Recuperado de: http://www.artsandarchitecture.com/index.html (09/08/2021)

Vela Castillo, J. (2003). Richard Neutra, un lugar para el orden: un estudio sobre la arquitectura natural. Sevilla: Universidad de Sevilla. 